\title{
Development of the Process Information Modelling in the construction project: A case study of the ZERO-PLUS project
}

\author{
Wen Pan ${ }^{\mathrm{a}}$, Katharina Langosch ${ }^{\mathrm{a}}$ and Thomas Bock ${ }^{\mathrm{a}}$ \\ ${ }^{a}$ Chair of Building Realisation and Robotics, Technical University of Munich, Germany \\ E-mail: wen.pan@br2.ar.tum.de, katharina.langosch@br2.ar.tum.de
}

\begin{abstract}
-
The conventional Building Information Models (BIM) does not contain knowledge about the relation between different pieces of information. To be able to understand the information the recipient must have sufficient knowledge within the given domain. When information cannot reach to the relevant party in real time or in advance, that will cause miscommunication and delays. At this point, the Process Information Modelling (PIM), which is a process-oriented modelling approach, provides a collaborative way of planning, designing, producing, assembling, and managing the repeatable deployment process. The main objective of PIM is to formulate a process oriented database platform that allows smooth data transfer, exchange, as well as promoting seamless and constant data sharing. Digital documentation, simulation, graphical and non-graphical data are produced progressively to support the decision-making process throughout the PIM application development stage. Thus, PIM embraces real-time data processing that helps to identify issues in advance, predict malfunction, reduce production time, and influence production cost.
\end{abstract}

At the moment, PIM is developed through three main phases which include design, production, and on-site assembly phase and represents a conceptual tool that provides a platform where stakeholders can access information in real-time and to make vital decisions in advance. By using a real scenario in the on-going EU Horizon 2020 research project ZEROPLUS, the first application of the PIM concept could be demonstrated on the Freescoo HVAC system, which will not only help the project team to structure detailed guidelines within the current project but will also lay a foundation for developing a fully functional PIM application in the future.

Keywords -

PIM; off-site manufacturing; planning; simulation

\section{Introduction}

In the construction industry, software-based models are used in particular for complex building projects in order to make the planning and execution more efficient and optimize. Through the use of the so-called Building Information Model (BIM) method, a flexible and timely data exchange can be achieved between all involved parties (e.g. architects, engineers, etc.) in particular, if changes need to be adapted. Despite the eminent work relief by the use of BIM, it mostly provides detailed information about each component and thus, it is a knowledge-based method. However, the exact processes and methods of manufacturing, installation and assembly cannot be depicted.

The concept of Process Information Modelling (PIM), which is a relatively new method, offers the possibility to include precisely these processes. When applied in the context of off-site manufacturing and assembly tasks, it serves as a tool that guides involved personnel or any other entities on making an appropriate response to a specific task.

For this reason, the overall objective of PIM can be described as following: an innovative process-oriented approach that can be implemented to simulate and optimize the design, planning, production, and assembly process within the construction industry. This objective is motivated by the belief that current BIM has many limitations, which only partially enhance the construction process. PIM will be designed, as a sufficient tool that can simulate, evaluate and implement varied phases which is needed in today's competitive market. However, due to the novelty of the concept and the complexity of the construction process, the paper focuses on the presentation of the PIM concept and its potential and demonstrates a simple process directly selected from the ZERO-PLUS project that PIM application applied to the Freescoo technology used in case study Cyprus to describe the underlying principle.

The research team will carry out evaluation and identification of the key research boundaries within the 
context of the ZERO-PLUS project. The proposed PIM will provide a theoretical solution that offers an extensive guideline for the design, planning, simulation and optimization of off-site production and assembly phases. As already mentioned, the conventional BIM application is a knowledge-based solution, while PIM offers a know-how-based solution. As a result, utilization of both solutions can yield significant benefits for an organization or individuals undertaking a variety of complex projects. In addition, the proposed PIM approach will not only help the project team to structure detailed guidelines within the ZERO-PLUS project but also lay a foundation for developing a fully functional PIM application for the construction industry.

\section{Concept of PIM}

PIM can be understood as a process-oriented modeling approach that provides a collaborative way of planning, designing, producing, assembling, reinforced by the key phases namely; design, production, and onsite assembly. The key phases are further evaluated via various modeling applications, which enable stakeholders to use more efficient methods to create added value as well to optimize an existing operation.

First, the fundamental feature of PIM is a processoriented information modeling approach. Processoriented means that this approach is focused on a certain cluster of the entire process. On an organizational level, process planning, management, and integration make possible the improvement of interdepartmental, crossfunctional, and cross-disciplinary interactions. The goal is to optimize the entire process rather than optimizing only parts of it and neglecting the others. On a taskbased level, this can be achieved by a thorough understanding of end-to-end process and production systems as a whole [1].

Second, within each process, there are objects and other parameters such as location, and time. Any change in these elements would affect the operational performance of the overall process. Within PIM, the information model defines the objects and all other relevant parameters depending on the specific process. PIM application has the potential to be developed as a software program. The product and the Off-site Manufacturing (OSM) facility's property, class, protocols and assembly method can be easily translated to pseudocodes, which can be operationalized by software engineers.

Third, the assembly evaluation method (AEM) evaluates the data generated by the PIM and then, according to the real facilities, suggests production and assembly solutions. The AEM can also forecast the productivity of the proposed facilities or method.

Fourth, PIM has the potential to offer automated instructions when a key parameter is altered. This is extremely beneficial when the responsible parties do not have the required domain knowledge or are struggling to make the final decision. At the time of this writing, PIM is only capable of offering basic instructions that do not lead to unnecessary complications or disputes.

\section{PIM application and potential impact}

In general, PIM application consists of the following stages: identification, data acquisition, organization, and implementation, (Figure 1Error! Reference source not found.).

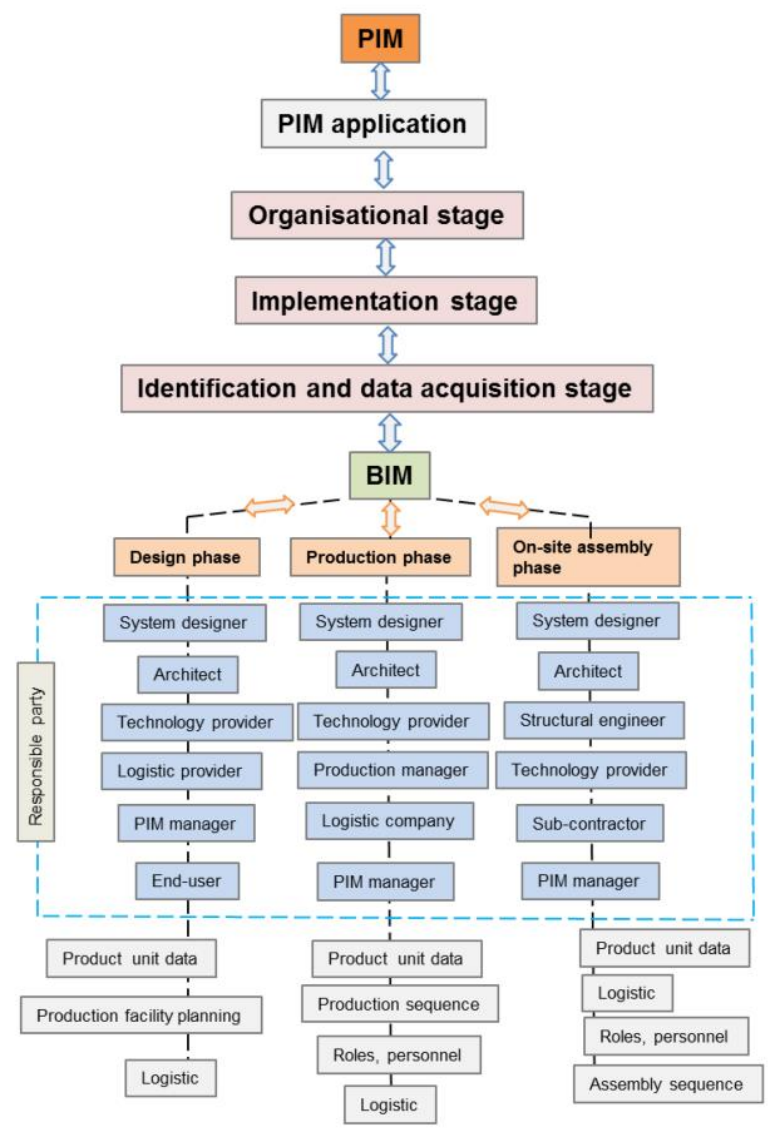

Figure 1: PIM chart

During the identification and data acquisition stage, the PIM manager will identify a number of parameters including the product definition, and the configuration of the product. The basic physical product features, dimensions, shape, material, functionalities and capabilities - are classified as unit data. Depending on the unit data and the production method, a manufacturing facilities concept will be proposed. The degree of automation will be applied in the production phase, as is often advised by the production manager, 
since it is directly influenced by other elements, such as the volume of the production, cost, demographic reasons and so forth. The logistic solution will be planned in advance to ensure Just-in-time (JIT) supply of parts and final products, both off-site and on-site [2]. The on-site assembly method is to be identified prior to delivery. Moreover, the product life-cycle management strategy is developed simultaneously to ensure future upgrade and maintenance. In summary, this stage performs a preliminary investigation the overall tasks.

During the organizational stage, based on the attributes of each identified unit data, the PIM manager will assign them to the corresponding phase. A class system will be applied to distinguish the attribute of each unit data. In doing so, a detailed representation of the affiliation between each unit data can be created. A detailed description of what needs to be accomplished at each sequence is produced. For instance, detailed product specification, alternation flexibility, material flow, tool and equipment being used, an alternative method, and additional requirements was determined based on the unit data. More importantly, at this stage, the PIM manager needs to be aware of the interconnection and independence of each unit data; when one of them changes, what would the trade-off and parallel impact on the others be? In summary, this stage performs planning, projecting and data arranging for the next stage.

During the implementation stage, the PIM manager will finalize each process and acquire a potential optimization strategy. For example, the final design of the product, the sequence of operations for manufacturing every part of the product, the required machinery, equipment, and personnel, the sequence of operations in the fabrication and assembly processes, smart buffering, packing station and loading method, supply and logistics strategy, on-site assembly or disassembly method and lifecycle management strategy. The data will serve as a guideline for different stakeholders.

In practice, PIM can alternatively be divided into a number of model fragments called information modules. The information modules contain dedicated information from each task phase. Typical task phases include design, production, logistic, on-site assembly, and lifecycle management. It is noticeable that these phases are also as part of the PIM application stage. The domain experts of each phase can function independently while also interactively working alongside the PIM manager to change and optimize the information module. For example, the product design phase is defined by the design brief of the product. The protocols between these phases are complementary: If the design engineer changes the product design, the PIM system keeps track of the changes, such that the other phases can respond to the changes accordingly.

\section{Usage of PIM in conjunction with BIM}

In recent years, there has been a shift from using $2 \mathrm{D}$ technical drawings to a more dynamic trend regarding the use of Building Information Models (BIM) within the architecture, engineering and the construction industries. Using modern modeling tools, such as Revit Architecture, ArchiCAD or Tekla Structures, the content produced by architects, designers and engineers have evolved from traditional 2D-drawings, sketches and written specifications to parametric, object-oriented 3D-models embedded with information to describe any building or facility in detail. As a digital representation of the physical and functional characteristics of a building, a BIM serves as a repository of information supporting a multitude of applications, including costestimation, energy analysis and production planning [3]. BIM covers the project span from the conceptual stage to the completion of the project. From construction scheduling to logistics management, BIM enables project managers and design-build teams to develop an object-oriented database that assists in predicting and measuring the project performance objectives. For construction management, BIM provides a virtual representation of the project concept prior to construction, ensures early failure detection, simulates and analyses potential impacts, and improves safety [4]. Moreover, BIM allows information transfer between all stakeholders, by allowing each stakeholder to contribute the information they acquire during the construction of the BIM system [5].

In comparison to PIM, BIM does not contain knowledge about the relation between different pieces of information. A piece of information cannot function alone but must coexist with other pieces. Even though BIM allows information sharing and transferring, this information is typically only available to parties that already have sufficient knowledge within the given domain. Consider a building contractor who would like to purchase a set of the prefabricated foundation. The BIM information about the prefabricated foundation will only contain the specification and compatibility of the product. The duration and the exact process and method of delivering and installing the foundation product are not included. In fact, the installation process contains the most crucial information for the project team preparing the installation tasks, in order to keep the project on schedule.

In contrast, PIM would provide detailed information on not only the specification and cost of the product, but also on detailed processing instructions for the installer, such as the depth of a trench, tools, and equipment required, and illustrations of connection methods to 
assist the team throughout the project phase. Judging by the example above, BIM represents what the product consists of, whereas PIM represents what the product consists of and, in addition, how it responds to the user's requirement and the methods used [6].

\section{Influence of PIM in manufacturing}

To develop the most relevant PIM application for the future construction industry, the following topics were studied and analyzed, which include, Lean manufacturing concept, Design for manufacturing, Justin-time (JIT) supply and assembly automation.

"Lean" is a term in the manufacturing sector that originated from the Toyota production system (TPS) [7] and the book named "Lean Thinking" by [8]. The lean manufacturing concept prioritizes eliminating waste during the production phase [9]. Since the beginning of the industrial revolution, manufacturers have tried to overcome the issue of how to eliminate waste. In this context, the waste refers to the unnecessary expenditure of time, money and materials [10]. The Muda concept is one of many concepts (Muda means 'waste' in Japanese), which focus on non-value added activities in terms of production. In total, there are seven types of Muda namely: overproduction, waiting (queueing), transportation, processing, inventory, motion, rework, and poor resource utilization. All of them can contribute a non-value added activity that will not improve the product [11]. Though Lean Manufacturing has been used in the Japanese construction industry for a long time, in Europe it has been adopted only in the last decades. In the future, PIM application can potentially reduce non-value added activities throughout the construction process and enhance communication between all project phases.

Design for manufacturing (DfM) is a concept that encourages designs that are easy to produce. It is closely associated with another concept called design for assembly (DfA). However, there is a fundamental difference between these two concepts. In general, DfM focuses on designing individual parts (product), whereas DfA focuses on the protocols between the products, in other words how to assemble complex products from integrated parts easily. The combination of these two principles is known as design for manufacture and assembly (DFMA). In the manufacturing sector, it is more feasible to change the design of the product rather than to alter the existing production facility. PIM is highly relevant in improving the DFMA concept that the information collected by PIM can be used to structure the protocols between the product and the production sequence, therefore enhance the overall manufacturing efficiency.

Just in Time (JIT) refers to the strategy developed first in the Toyota production system to minimize the space needed to supply the production phase. Usually mentioned together with Just in Sequence (JIS), it has been identified as one of the main success factors of the company. In the construction field, the JIT model can be used not only for off-site, usually largely depending on the level of prefabrication employed, but also for on-site activities. This is because the on-site assembly of prefabricated elements requires an amount of time, resources, and attention is usually greater than for offsite operations. This is due to unpredictable and unexpected external circumstances. PIM can potentially help the construction project team to synchronize offsite and on-site operations together with JIT model [12].

Generally, automation substitutes human activity in difficult and repetitive tasks, thus enhancing productivity and safety, while considerably reducing the labor cost. The use of machines ensures that the quality of the final product remains at a consistent level, as robots reduce human error and the drawbacks derived from hostile weather conditions. Moreover, the planning phase is also enhanced, due to the high predictability of the process [13]. The level of assembly automation in an off-site construction plant varies considerably depending on several factors, such as the material and the complexity of the product. Nonetheless, it is generally advantageous to achieve as high level of automation as possible, in order to fully enhance production and obtain a quick return on investment. In this paper, the outcomes that influenced by using PIM application during the design, automation in production, assembly of the Freescoo HVAC system will be briefly described.

\section{PIM application in ZERO-PLUS}

One of the goals of the ZERO-PLUS project is to develop sustainable buildings and to minimize their Embedded Energy, especially concerning the manufacturing stage. To employ a zero-waste strategy, the project combines Lean Construction off-site manufacturing. The different aspects reviewed here can be all used in a synergistic way to achieve a modular, sustainable, productive and cost-effective system. Regards to the ZERO-PLUS project, PIM is used to simulate and optimize the planning, production, and assembly phase. In the project, the Freescoo HVAC system used in the Cyprus case study provides the most applicable example to demonstrate the potential of implementing a PIM application in the real scenario. In terms of process management, the project is divided into four task phases, which include design, production, logistics and on-site assembly, (Figure 2).

During the design phase, the features of the proposed frame for the Freescoo HVAC system were 
analyzed. By conducting an analysis of the unit data, the production method was determined, allowing the design engineers to map out the optimum production sequence. The manufacturing facility was also strategically designed. In order to examine the protocols between the product and production design phase, three different frame designs are presented. Consequently, the differences in each frame design will present a variation in its production as well as in the assembly phases.

During the production design phase, a step-by-step procedure of the process using tables, graphics, and simulation creates an accurate representation of what will happen in the real situation. A set of goals was clarified to achieve a successful production facility design. In addition, the product unit data was also considered, as it will directly influence the sequence of assembly, workflow analysis, production plant specification, degree of automation, the number of machines needed, material flow and handling method, the activity relationship between each task, etc. An agent class system assigns each task a reasonable role [14], which can be a person, machine, or a robot that will perform the given task. Initially, the agent class will be documented, but later the data has the potential to construct a software algorithm that assists PIM manager in filtering data, interpreting information, monitoring activities, and making decisions. Agents in PIM application domains exhibits four key characteristics, i.e. autonomy, reactivity, communication and goal orientation. They are capable of acting autonomously, cooperatively, and collectively [15]. By using its agent class, PIM systematically represents the dynamic relationships between tasks. Moreover, during this phase, the proposed AEM was developed in parallel to evaluate the difference in productivity caused by the variations in the frame designs.

The On-site assembly phase focuses on the case study in Cyprus. The structural performance of the building was studied. To achieve smooth execution, the architects and assembly engineers received a detailed assembly plan according to the PIM data collected through the project phases.

By using the proposed PIM method, each project team role will be able to access the relevant information in advance or in real time. Data transfer and sharing between different domains facilitate the project team's understanding of the actual process and the method required to accomplish the task. The development of the AEM in conjunction with PIM can be used as a decision support tool to manage sources of complexity and rationalize the changes of product features, the unit data, and design alternatives to proactively response to the decision-making process.

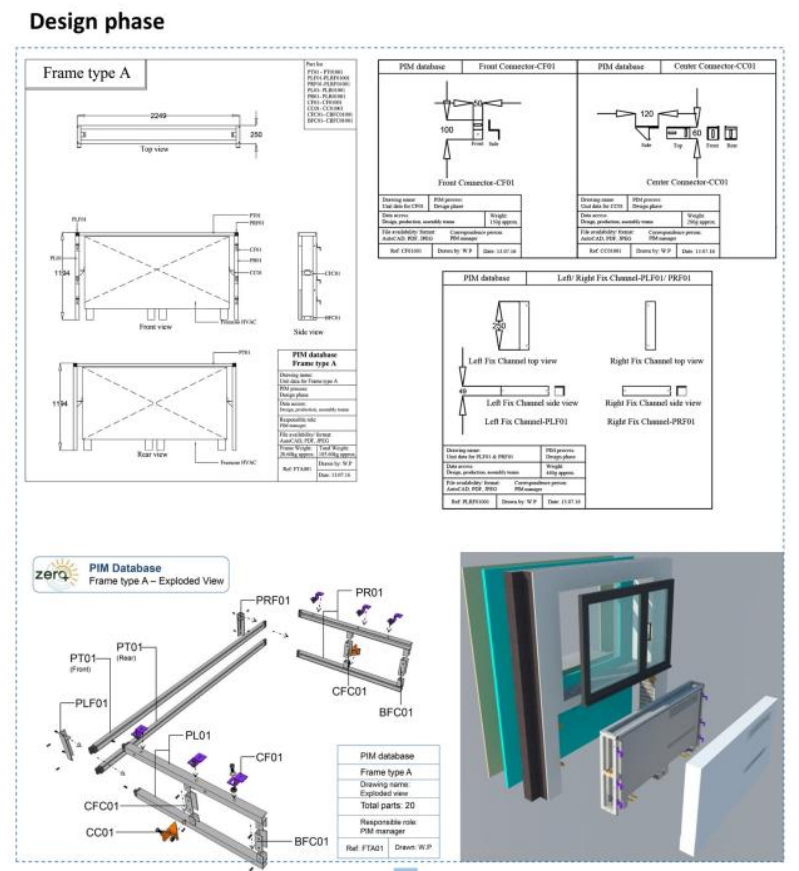

Production design phase

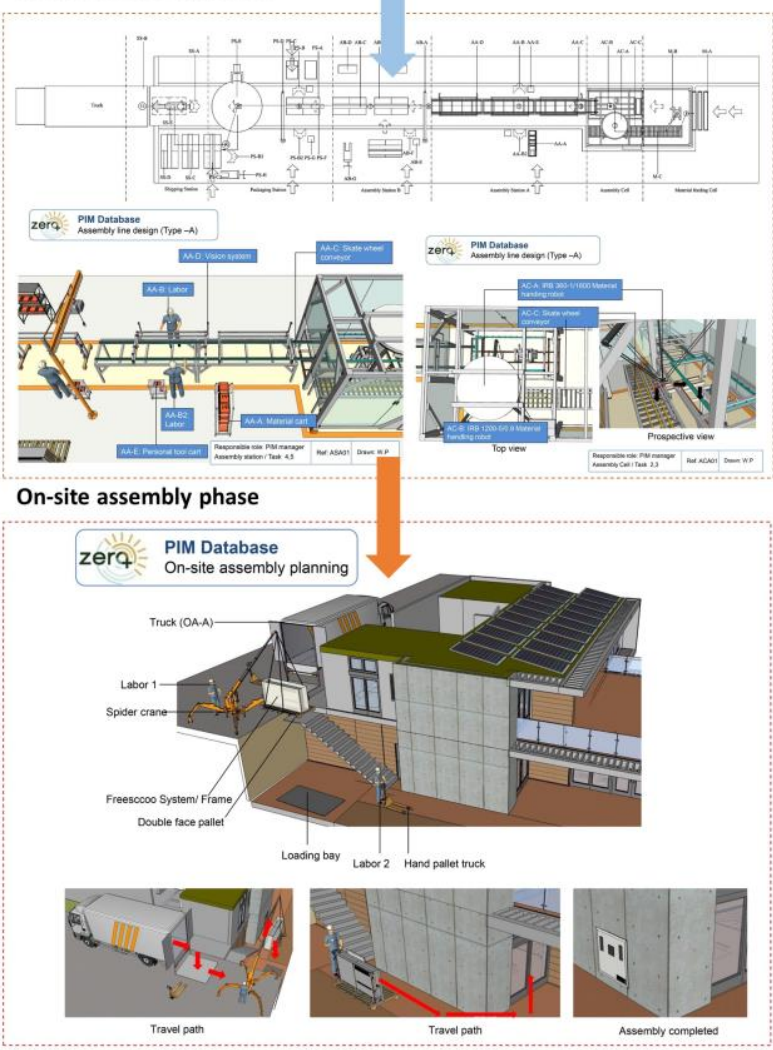

Figure 2: PIM application in the ZERO-PLUS project include design, production, on-site assembly phase 


\section{Brief description of the design of the integrated, flexible frame system for the Freescoo HVAC system}

In order to fulfill the design goal from the project partner, the frame is designed as modular steel profile parts. The size and the shape of the frame are designed to ease handling by robots during assembly according to its kinematics and degree of the freedom (DOF). The proposed methodology inspired by the notion of DfX and Robot-Oriented Design (ROD) [16] incorporates the integrated assessment of parts and the evaluation of part complexity and accessibility. There is no welding applied at any joints to ensure maximum flexibility. Once the frame is installed, the design has to ensure that the Freescoo HVAC system is accessible in case of future maintenance or system upgrades. The PIM application in the project includes, generates unit data (e.g. specifications) of the frame, provides data to help the production design engineers map out the applicable production sequence, and the method to be used during the production phase. In addition, PIM provides data to assist the architect in altering the building design to compatible with the proposed frame and provide a guideline that will assist the on-site assembly procedure.

\section{Optimization of PIM application}

The ZERO-PLUS project provides a real-world example that demonstrate the theoretical potential of the PIM application in dealing with OSM strategy, assembly process, and management of the construction project. The research also serves as a guideline for the PIM initial application development stage in which recommended methods will accumulate and register essential data, as well as classifying different agents. The experience gained from the ZERO-PLUS project will act as the foundation for the future optimization of PIM. The next phase of PIM application development will focus on the design of the program architecture that will interpret the data collected from a generic software application in the initial stage, to ensure further implementation in a much broader range of projects.

\section{Future software / computer application}

During the development of PIM concept, the research team realises that there are various examples in computer software design, which have conceptual principles similar to those in PIM. A brief analysis is conducted to evaluate the state-of-the-art trends in software engineering, and to offer a reinterpretation of PIM by using software-programing concepts.

Service-oriented architecture (SOA) can be described as a loosely coupled program architecture designed specifically to meet the needs of an organization. By using communication protocols which provide services to another component, and make connections between different software components over a network [17], the definition of a service can be viewed as a logical representation of a repeatable task. SOA is independent, self-contained, yet when combined it forms the functionality of a large software application. Service orientation provides a dynamic, collaborative application for software integration and task composition. The capacities of a PIM architecture can be influenced by SOA. In PIM, the unit is similar to a service which is unrelated, self-contained, and implements one or more actions. The unit architecture in PIM also shares similarities with the service architecture in SOA [18]. It is beneficial when linking an agent influenced by the unit data or service, and any alteration of the unit or service would influence the individual agent's capabilities or responsibilities.

Service discovery or service discovery protocols (SDP) is an emerging field in the area of ubiquitous computing. They provide a mechanism, which allows automatic detection of service offered by any node in the network. In other words, service discovery is the action of finding a service provider for a requested service [19]. Service discovery can serve as a search engine in PIM architecture. For example, when the technology provider uploads a new product to the PIM database, if the specification matches with any user's requirements, the system will recommend the product automatically.

Microservices is a relatively new concept in the software design domain. It inherits the basic characteristics of service-oriented architectures (SOA). Conventionally, the program is a monolithic architecture with limited flexibility. Large complex software applications are composed of a number of services. Microservices offer solutions that can be deployed independently, specifically tailored, and loosely coupled [20]. The characteristics of the Microservices have served as an important source of inspiration in the development of the PIM concept. In practice, the assembly process can be considered a continuously deployed process. By adopting a Microservices architectural style in PIM, it enables the project teams to easily manage multiple aspects of the project. When issues arise during production, it is straightforward to pin down problems simply by identifying the smaller non-responsive Microservice processes or reviewing log files registered with a Microservice team identifier. Once the issue is identified, the responsible team member will be brought in to troubleshoot the problem [21]. 


\section{Variation and alternatives}

After an overview of the potential contributions from the software engineering sectors, the following section presents variations of the PIM application which could be implemented in the future in the construction industry. PIM shows great potential for the optimization of the assembly and production phases when applying the same principles. It is also suitable for other applications in the construction industry. For future reference, in this case, the PIM data module is divided by number of tasks include, (Figure 3).

1. PIM application for manufacturing of the building components.

2. PIM application for the building component attributes management.

3. PIM application for supply logistics.

4. PIM application for on-site assembly.

5. PIM application for home appliance management.

6. PIM application for green home appliances.

7. PIM application for service management.

8. PIM application for building performance.

9. PIM application for smart home environment control.

Because PIM can be loosely coupled, it can be specifically developed for each task. The loose coupling enables greater system flexibility that allows frequent rapid deployment.

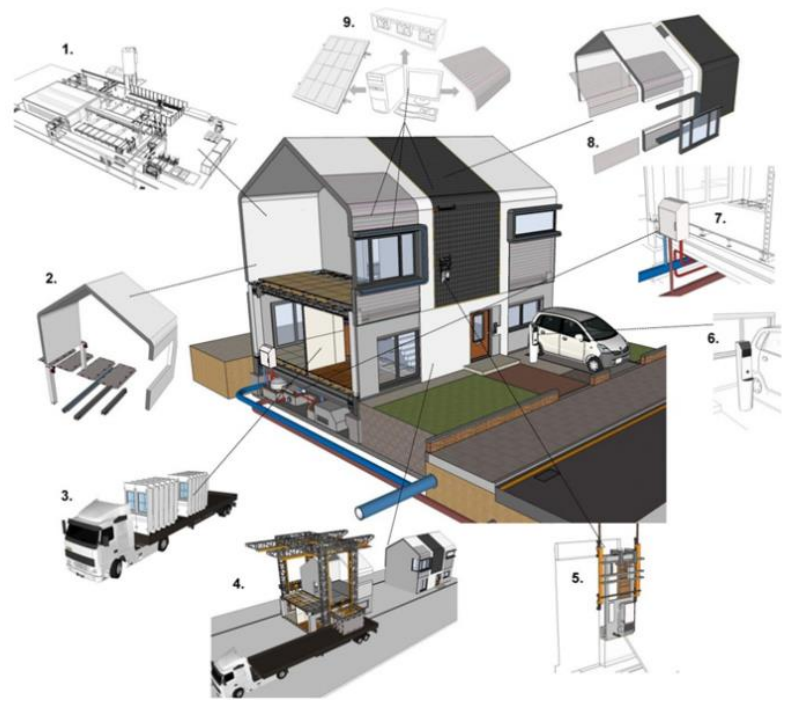

Figure 3: Future potential applications

\section{Conclusion}

During the ZERO-PLUS project, the research team gains an opportunity to develop the PIM concept that based on the Freescoo technology used in the Cyprus case study to demonstrate the potential of the PIM application in dealing with OSM strategy, assembly process, and management of the construction project. For now, PIM functions as a conceptual documentation tool. As mentioned earlier, PIM can be described as an interpretation of a process-oriented modeling approach, reinforced by the key parameters or rules, which are embedded within the operational process. When developing PIM as a software program, first, a pseudocode need to be developed to help the software engineer to understand the proposed PIM architecture. Then, detailed and exclusive information is needed to development a concrete PIM database. The PIM database and the pseudocode will generate the processing logic that assists the software engineer in further developing PIM as a functional software program. However, in terms of future implementation in industry, there are still many challenges ahead. Effective communication between the PIM manager and the software engineers is required. A real case scenario is necessary to collect the relevant information for database development. The hardware and software environments required for PIM to function as a universal application have not yet been studied.

\section{Acknowledgements}

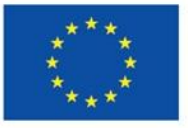

This project has received funding from the European Union's Horizon 2020 Research and Innovation programme under Grant Agreement No. 678407.

\section{References}

[1] Schweitzer, E., \& Aurich, J. C. Process Oriented Production System for Service Providing Companies. In Proceedings of the $2^{\text {nd }}$ CIRP IPS2 Conference 2010; 14-15 April; Linköping; Sweden (No. 077, pp. 323-329). Linköping University Electronic Press, 2012.

[2] Battini, D., Boysen, N. and Emde, S. Just-in-Time supermarkets for part supply in the automobile industry. Journal of Management Control, Vol. 24 No. 2, pp. 209-217, 2012.

[3] Eastman, C., Teicholz, P., Sacks, R., Kathleen, L., \& Handbook, B. I. M. A Guide to Building Information Modeling for Owners, Managers, Designers, Engineers and Contractors, Hoboken, 2008.

[4] Zhang, L. and Issa, R.R. Ontology-based partial building information model extraction. Journal of Computing in Civil Engineering, 27(6), pp.576- 
$584,2012$.

[5] Huang, C. J., Trappey, A. J., \& Yao, Y. H. Developing an agent-based workflow management system for collaborative product design. Industrial Management \& Data Systems, 106(5), 680-699, 2006.

[6] Dossick, C. S., \& Neff, G. Organizational divisions in BIM-enabled commercial construction. Journal of construction engineering and management, 136(4), 459-467, 2009.

[7] Ohno, T. Toyota production system: beyond largescale production. crc Press, 1988.

[8] Womack, J. P., Jones, D. T. Lean Thinking. Banish Waste and Create Wealth in Your Corporation, Revised and Updated. Simon \& Schuster, Inc, 2003.

[9] Selçuk, B. Adaptive lead time quotation in a pull production system with lead time responsive demand. Journal of Manufacturing Systems, Vol. 32 No. 1, pp. 138-146, 2013.

[10] Diekmann, J., Krewedl, M., Balonick, J., Stewart, T., Won, S. Application of Lean Manufacturing Principles to Construction. University of Texas at Austin, 2004.

[11] Stephens, M. P., \& Meyers, F. E. Manufacturing facilities design and material handling. Purdue University Press, 2013.

[12] Javadian Kootanaee, A., Babu, K.N. and Talari, H.F. Just-in-time manufacturing system: from introduction to implement, 2013.

[13] Boothroyd, G. Assembly automation and product design (Vol. 536). Boca Raton, FL: Taylor \& Francis, 2005.

[14] Turban, E., King, D., Lee, J. and Viehland, D. Electronic Commerce: A Managerial Perspective, Pearson Education International, 2004.

[15] Parker, H. Interfirm collaboration and the new product development process. Industrial Management and Data Systems, Vol. 100 No. 6, pp. 255-60, 2000.

[16] Bock, T. Robot-oriented design. Proceedings of the $5^{\text {th }}$ ISARC, Tokyo, Japan, pp. 135-144, 1988.

[17] Erl, T. Service-oriented architecture: a field guide to integrating XML and web services. Prentice Hall PTR, 2004.

[18] Patrick, P., Aletty, A., Kasi, J., Kapoor, C., Urhan, T. and Mihic, M. Bea Systems, Inc. Service oriented architecture. U.S. Patent Application 11/133,022, 2005.

[19] Czerwinski, S. E., Zhao, B. Y., Hodes, T. D., Joseph, A. D. and Katz, R. H. An architecture for a secure service discovery service. In Proceedings of the $5^{\text {th }}$ annual ACM/IEEE international conference on Mobile computing and networking (pp. 24-35). ACM, 1999.
[20] Balalaie, A., Heydarnoori, A., Jamshidi, P. Microservices Architecture Enables DevOps: Migration to a Cloud-Native Architecture. IEEE Software 33 (3): 42-52, 2016.

[21] Chen, L. and Babar, M. A. Towards an evidencebased understanding of emergence of architecture through continuous refactoring in agile software development. In Software Architecture (WICSA), 2014 IEEE/IFIP Conference on (pp. 195-204). IEEE, 2014. 